\title{
Clostridium difficile tcdC DNA Measurement
}

National Cancer Institute

\section{Source}

National Cancer Institute. Clostridium difficile tcdC DNA Measurement. NCI Thesaurus. Code C150861.

The determination of the amount of tcdC DNA produced by Clostridium difficile present in a sample. 